\title{
Granulocytic sarcoma of the breast in acute myeloid leukemia: Two case reports
}

\author{
JIANFEI FU and JIANSHENG LUO \\ Department of Oncology, Jinhua Central Hospital, Jinhua, Zhejiang 321000, P.R. China
}

Received April 19, 2013; Accepted October 28, 2013

DOI: $10.3892 / \mathrm{ol} .2013 .1687$

\begin{abstract}
Granulocytic sarcoma (GS) of the breast is extremely rare in patients with acute myeloid leukemia (AML) and therefore, is often misdiagnosed as lymphoma or other benign tumors. The current report presents two cases of GS of the breast, of which, one was considered to be a fibroma, as observed by fine-needle aspiration, and the other was misdiagnosed as lymphoma by frozen section. Previous literature that described the clinical and pathological characteristics, treatments and prognosis of GS of the breast in AML were reviewed. In addition to the treatment of mastectomy with/without radiotherapy, lumpectomy may also be received as a good treatment plan.
\end{abstract}

\section{Introduction}

Granulocytic sarcoma (GS) is a solid tumor containing myeloid leukemia cells that appears in a variety of locations, including the central nervous system, bone and soft tissue. To the best of our knowledge, GS invading the breast is rare (1) and may present at the onset of acute myeloid leukemia (AML) or extramedullary relapse following bone marrow transplantation. No characteristic appearances of GS of the breast have been identified by radiography or ultrasonography. In addition to its rarity, GS of the breast is easy to misdiagnose as other benign tumors, lymphoma or sarcoma. Currently, the only method to confirm the diagnosis of GS of the breast is by pathological examination combined with immunohistochemistry. Doctors must take GS of the breast into consideration when the diagnosis is confusing. The standard therapeutic approach for GS of the breast remains undefined; therefore, doctors face a dilemma in making a clinical decision. The current report presents two cases of GS of the breast and a review of the literature. This study was approved by the Ethics Committee

Correspondence to: Dr Jianfei Fu, Department of Oncology, Jinhua Central Hospital, 351 Mingyue Street, Jinhua, Zhejiang 321000, P.R. China

E-mail:nuifu@sina.com

Key words: breast, granulocytic sarcoma, myeloid leukemia of Jinhua Central Hospital and was performed according to the Declaration of Helsinki. Written informed consent was obtained from each patient's family.

\section{Case reports}

Case 1. A 59-year-old female presented on June 25, 2008, with a painless mass in the left breast that had been present for one week and a fever with a temperature of $37.0-37.7^{\circ} \mathrm{C}$. The patient exhibited no other symptoms or relevant past or family histories. A physical examination revealed a mobile mass in the left breast measuring $1.8 \times 1.2 \mathrm{~cm}$, with no palpable auxiliary lymph nodes.

Ultrasound revealed a solid and hypoechoic mass measuring $1.7 \times 0.9 \mathrm{~cm}$, which had a clear boundary with hypervascular flow, and the mammography showed an equidensity mass with no areas of calcification. The chest radiography revealed no abnormal observations, and abdominal ultrasound revealed no suspected lesions in the liver or spleen. The complete blood count showed a white blood cell (WBC) count of $2.9 \times 10^{9} / 1$, a neutrocyte count of $0.2 \times 10^{9} / 1$, hemoglobin levels of $109 \mathrm{~g} / 1$ and a platelet (PLT) count of $104 \times 10^{9} / 1$.

Since the mass in the patient's breast was considered to be a benign tumor, a lumpectomy had to be performed under local infiltration anesthesia. The patient underwent the lumpectomy on June 10, 2008, despite a relatively low WBC count. According to the frozen section, the patient was diagnosed with breast mucosa-associated B-cell lymphoma. Post-operative pathology revealed that the nuclei of the tumor cells were evidently different in size and shape and scattered among the massive fibrous tissue. At the edge of the tumor, the tumor cells exhibited a streamline alignment (Fig. 1A) and mitotic figures were easily identified. The immunohistochemical analysis showed that markers of myeloperoxidase (MPO) and CD45RA were positive and those for carcinoembryonic antigen (CEA), L26 and CD3 were negative, while CD45RO was weakly positive (Fig. 1B). The patient was confirmed with AML-M4E0 by bone marrow aspiration, and the final diagnosis was GS of the breast.

The patient received inducted chemotherapy with the MA regimen (i.v. injection of $6 \mathrm{mg} / \mathrm{m}^{2}$ mitoxantrone days $1-3$ plus i.v. injection of $100 \mathrm{mg} / \mathrm{m}^{2}$ cytarabine day $1-3$, every 3 weeks) and achieved complete remission. The patient subsequently received consolidation chemotherapy. To date, the patient has received follow-up for 4 years and no relapse has occurred. 
Case 2. A 37-year-old female was diagnosed with AML-M6 by bone marrow aspiration and biopsy in April, 2004. The patient achieved complete remission following inducted chemotherapy with retinoic acid $\left(45 \mathrm{mg} / \mathrm{m}^{2}\right.$, days $\left.1-28\right)$. Following a total of 8 cycles of consolidation chemotherapy with the MA regimen, the patient underwent allogeneic bone marrow transplantation in January, 2006.

In November 2006, the patient was admitted to the Department of Oncology (Jinhua Central Hospital, Jinhua, China) due to a painless palpable mass in the left breast that had been present for 10 days. The patient exhibited no other symptoms and had no relevant previous or family histories. A physical examination revealed a mass measuring $2.0 \times 1.5 \mathrm{~cm}$ in the lower inner quadrant of the left breast. Ultrasound revealed a solid and hypoechoic mass, and fine-needle aspiration (FNA) identified the mass as a fibroma. No suspicious lesions were located in the patient's lungs by chest radiography or in the liver by ultrasound.

The complete blood count showed a WBC count of $2.9 \times 10^{9} / 1$ with $53.4 \%$ neutrocytes, a red blood cell count of $1.94 \times 10^{12} / 1$ with $72.0 \% \mathrm{HCT}$ and a PLT count of $27 \times 10^{9} / 1$. Since the mass was diagnosed as a benign tumor by FNA, the patient underwent a lumpectomy under local infiltration anesthesia, despite having a low PLT count. The tumor was removed in November, 2006, and the pathological results revealed that the structure of the breast, which had been invaded by middle-sized diffuse lymphoid cells and fat tissue, was destroyed (Fig. 2A). Immunohistochemical analysis revealed that the markers of MPO and LCA were positive and that those for CD68, CD79a and L26 were negative (Fig. 2B). Following confirmation by histopathology, the patient was diagnosed with GS of the breast. The tests for liver function, performed in February, 2007, demonstrated that the levels for alanine aminotranferase, aspartate aminotransferase, total bilirubin, direct bilirubin and r-glutamyltransfarase were $97 \mathrm{IU} / 1,186 \mathrm{IU} / \mathrm{l}, 90.7 \mu \mathrm{mol} / \mathrm{l}$, $40.8 \mu \mathrm{mol} / 1$ and $1,064 \mathrm{U} / 1$, respectively, with normal reference values of 0-40 IU/1, 0-40 IU/1, 2-25 $\mu \mathrm{mol} / 1,0-8 \mu \mathrm{mol} / 1$ and $0-50 \mathrm{U} / 1$, respectively. The level of the immunoglobulin IgG protein subtype was $4,240 \mathrm{mg} / \mathrm{ml}$ (normal reference value, $723-1,685 \mathrm{mg} / \mathrm{ml}$ ). The patient was diagnosed with graft-versus-host-disease (GVHD) and received immunosuppression therapy to protect the liver. However, the effect of the treatment was unsatisfactory and the patient succumbed to fungal pneumonia in June, 2007, without having received systemic chemotherapy or a second bone marrow transplantation. The relapse of the GS in the patient's breasts was not identified prior to mortality.

\section{Discussion}

The prevalence rate of GS is $~ 8 \%$ of all AML cases (1). GS may occur simultaneously with leukemia or as the initial symptom of recurrence following bone marrow transplantation in patients with AML. The mechanism of GS remains undefined and no definite associations between GS and bone marrow transplantation have been identified. The French-American-British classification subtypes of AML, M4 and M5, and a high WBC count may be predictors of GS (2). However, in the current report, case 1 was diagnosed with M4 and case 2 was diagnosed with M6, and a high WBC count

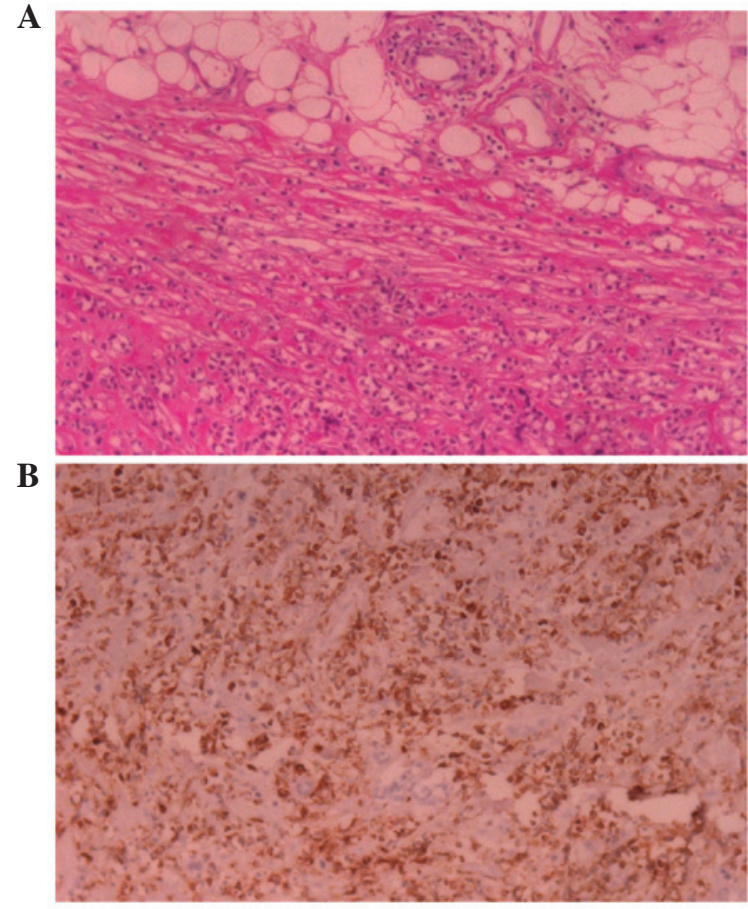

Figure 1. (A) In case 1, the normal breast structure was destroyed. At the edge of tumor, tumor cells were compressed by massive fibrous tissue into a streamline alignment. (B) Immunohistochemistry stain showing that MPO was markedly positive in the cell plasmid. (Hematoxylin and eosin staining; magnification, $\mathrm{x} 200$ )

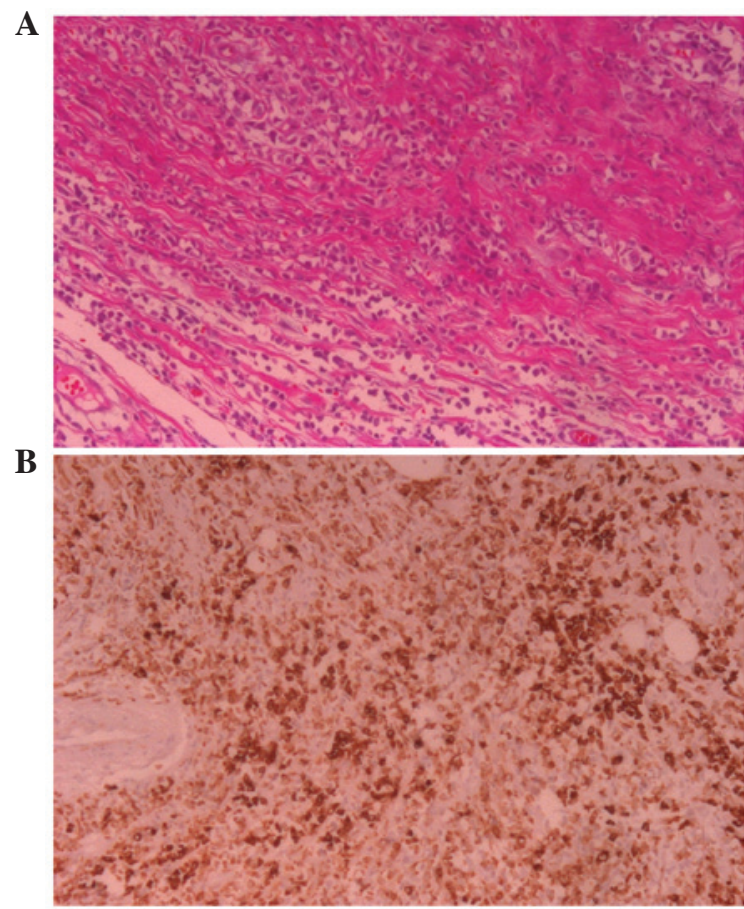

Figure 2. (A) In case 2, mesenchymal tissues were packed into the tumor cells, arranged into a line and the fatty tissue was infiltrated at the edge of the tumor. (B) Immunohistochemistry stain showing that MPO was markedly positive in the cell plasmid. (Hematoxylin and eosin staining; magnification, $\mathrm{x} 200$ ).

was not identified in either case, while a low WBC count was identified in case 1 . The results are inconsistent with the literature (2) and therefore, further study is required to detect 
predictors of GS. The mechanism of extramedullary relapse following allogeneic bone marrow transplantation remains unclear. There have been numerous cases of extramedullary relapse and normal donor cells in the bone marrow and it is possible that extramedullary relapse results from the graft-versus-leukemia (GVL) effect $(3,4)$. The graft induces the remaining leukemia stem cells in the marrow to release into extramedullary sites. The patient in case 2 was diagnosed with GVHD, and a previous study demonstrated that GVHD and GVL have the same mechanism (5). However, no reports have analyzed the correlation between GVHD and the extramedullary relapse of AML.

For patients with GS of the breast, the isolated nodule, multiple nodules or mass may be observed in the unilateral or bilateral breasts of patients of any age, even with a diffuse lesion. Patients do not present specific signs, including nipple inversion or discharge (6) and do not have a family history of breast cancer. The two cases in this report presented with unilateral isolated nodules. The ultrasound observations for GS of the breast are absent of characteristic appearances and often present as a hypoechoic and well-defined mass, with color flow imaging and without areas of calcification (7). Few case reports have previously described the mammographic appearance of GS of the breast $(8,9)$. GS has been commonly described as a non-calcified mass in the breast. In case 1 , the mammogram also presented a non-calcified, irregular mass, consistent with studies in the literature (10). The absence of characteristics in the imaging for GS results in the difficulty of differentiating GS from other breast diseases. Therefore, pathological analysis is necessary in order to confirm the diagnosis of GS. However, without sufficient preparation, due to the rarity of GS of the breast, the condition is usually misdiagnosed as a benign tumor or primary carcinoma of the breast by FNA, as was evident in the two cases of the current study. A biopsy is the only method to confirm the final diagnosis of GS, however, hematoxylin and eosin (H\&E) staining may reveal various morphological changes, resulting in the common misdiagnosis of GS as lymphoma or sarcoma (9). H\&E staining may reveal tumor cells that vary in size and with evident nuclear atypia (11). At the edge of the tumor, the vast mesenchymal tissue compresses the tumor cells into a streamline alignment. The immunohistochemical detection of MPO-positive cells is useful in order to confirm the final diagnosis, and primary breast carcinoma may be ruled out by the detection of cytokeratin-negative cells. B cell and T cell markers may be useful as further indicators for ruling out the diagnosis of lymphoma. By combining the history of AML with observations made in a bone marrow aspiration or biopsy, GS of the breast may be confirmed.

The therapeutic approaches for GS of the breast remain unconfirmed and there have been controversial opinions with regard to the local treatment of the breast. Mastectomy or lumpectomy with or without radiotherapy may be acceptable as treatments, and radiotherapy has been recommended in specific previous studies, however, the role of surgery remains unclear (12). The two cases presented in the current report were treated with lumpectomy alone and the results of local recurrence in the breast were satisfactory, as no relapse was identified in either of the patients. A lumpectomy may be an alternative treatment to consider for breast masses in such patients. When considering systemic therapy, untreated patients must receive bone marrow transplantation following induced chemotherapy, and patients with extramedullary relapse without bone marrow relapse may undergo strict observation without aggressive systemic treatment. However, when bone marrow relapse occurs, patients must receive systemic chemotherapy and/or a second bone marrow transplantation (9). Case 2 of the current report was diagnosed with GVHD and presented with GS of the breast as the initial symptom following allogeneic bone marrow transplantation. This case is the first such case to be reported and therefore, there were no successful treatments to refer to. Patients diagnosed with extramedullary relapse concomitant with GVHD commonly exhibit liver and renal dysfunction, which reduces the chance of receiving aggressive chemotherapies or a second bone transplantation.

The prevalence of GS of the breast in AML is rare, and a lumpectomy may achieve satisfactory local control and be an alternative treatment for solitary unilateral nodules in the breast.

\section{References}

1. Liu PI, Ishimaru T, McGregor DH, Okada $\mathrm{H}$ and Steer A: Autopsy study of granulocytic sarcoma (chloroma) in patients with myelogenous leukemia, Hiroshima-Nagasaki 1949-1969. Cancer 31: 948-955, 1973.

2. Byrd JC, Edenfield WJ, Shields DJ and Dawson NA Extramedullary myeloid cell tumors in acute nonlymphocytic leukemia: a clinical review. J Clin Oncol 13: 1800-1816, 1995.

3. Firas AS, Demeckova E, Bojtarova E, Czako B, Hrubisko M and Mistrik M: Isolated extra-medullary relapse of acute leukemia following allogeneic bone marrow transplantation. Bratisl Lek Listy 109: 358-361, 2008.

4. Karbasian-Esfahani M, Wiernik PH, Yeddu M and Abebe L: Leukemic infiltration of the breast in acute lymphocytic leukemia (ALL). Hematology 13: 101-106, 2008.

5. Koca E, Goker H, Guven GS, et al: Unusual extramedullary recurrences and breast relapse despite hepatic GVHD after allografting in Ph+-ALL. Hematology 11: 105-107, 2006.

6. Thachil J, Richards RM and Copeland G: Granulocytic sarcoma - a rare presentation of a breast lump. Ann R Coll Surg Engl 89: W7-W9, 2007.

7. Yang WT, Muttarak M and Ho LW: Nonmammary malignancies of the breast: ultrasound, CT, and MRI. Semin Ultrasound CT MR 21: 375-394, 2000.

8. Khoury NJ, Hanna Al-Kass FM, Jaafar HN, Taher AT and Shamseddine AI: Bilateral breast involvement in acute myelogenous leukemia. Eur Radiol 10: 1031, 2000.

9. Shea B, Reddy V, Abbitt P, Benda R, Douglas V and Wingard J: Granulocytic sarcoma (chloroma) of the breast: a diagnostic dilemma and review of the literature. Breast J 10: 48-53, 2004.

10. Pettinato G, De Chiara A, Insabato L and De Renzo A: Fine needle aspiration biopsy of a granulocytic sarcoma (chloroma) of the breast. Acta Cytol 32: 67-71, 1988.

11. Traweek ST, Arber DA, Rappaport H and Brynes RK: Extramedullary myeloid cell tumors. An immunohistochemical and morphologic study of 28 cases. Am J Surg Pathol 17: 1011-1019, 1993.

12. Mandal S, Jain S and Khurana N: Breast lump as an initial manifestation in acute lymphoblastic leukemia: an unusual presentation. A case report. Hematology 12: 45-47, 2007. 